\title{
Modelling of protective potential distribution in a cathodic protection system using a coupled BEM/FEM method
}

\author{
A. Muharemović ${ }^{1}$, H. Zildžo ${ }^{1} \&$ E. Letić ${ }^{2}$ \\ ${ }^{1}$ Department of Electrical Engineering, University of Sarajevo, \\ Bosnia-Herzegovina \\ ${ }^{2}$ Energoinvest d.d. Sarajevo, Bosnia-Herzegovina
}

\begin{abstract}
Design of any cathodic protection (CP) system requires one to determine current density which meets predefined criteria, and to ensure as uniform a current density distribution as possible over the structure surface. Nowadays the only reliable way to determine current and potential distribution is employment of numerical techniques. In this paper, the optimisation of a passive cathodic protection system by using coupled BEM/FEM is presented. FEM is appropriate for solving completely bounded domains, and it is used for discretization of anodes and cathodes. BEM is applied for discretization of electrolyte, i.e. infinite domain. In the coupled BEM/FEM the soil domain is linked to the steel domain through the current density (boundary condition) generated by the kinetics of the corrosion reactions at the steel surface. Verification of the obtained numerical result is made by comparing it with measured results. Good agreement between simulation and measurements has been found.
\end{abstract}

Keywords: cathodic protection, finite element method, boundary element method, galerkins weighted residuals method, successive underrelaxation method.

\section{Introduction}

The distribution of protection potentials in systems of cathodic protection (electrostatic field with or without sources) is defined either by the Poisson or Laplace equation, with the usage of corresponding boundary conditions. 
Boundary conditions in the cathodic protection system, in most cases, are not linear because the electrochemical reactions do not give linear functional relations between the protection current density and the corresponding potential. This piece will treat nonlinear functional relations $V=f(\sigma)$ and the case of a homogeneous electrolyte, i.e. electrolyte with the same average value of soil resistivity [2].

On the figure 1 the basic model of cathodic protection is given. The galvanic anode is made of cast zinc. Positive ions from the anode move through the electrolyte towards the object that is being protected, while electrons move in the opposite direction. DP (drain point) represents the point where the cable installation connects with the object being protected - the pipeline.

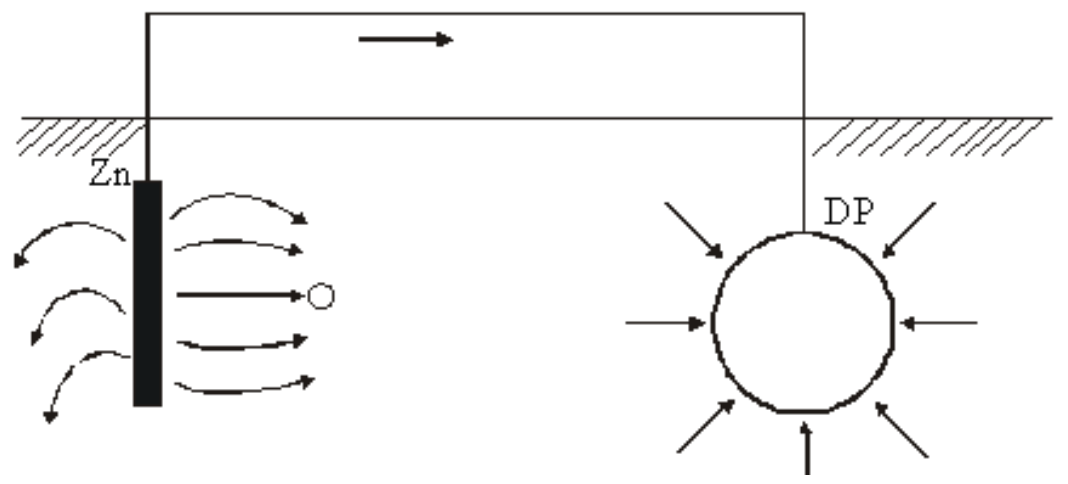

Figure 1: $\quad$ Cathodic protection system with galvanic anodes.

The authors have developed software for modeling and optimization of cathodic protection systems with galvanic anodes. Idea for this project is based on the works in field of cathodic protection that Bosnian company Energoinvest carried out in Libya in period 2003-2005 year. Owner and investor of the works was Great Man-made River Authority (GMMRA). The contractual obligation was to protect cathodically a pipeline in total length of $204 \mathrm{~km}$, and with internal diameter of 4 meters. All the measured results before and after CP system installation were available and these results enable the authors to make software validation i.e. comparison measurements and numerical calculation outcome.

\section{CP system design}

The system was designed to provide a $250 \mathrm{mV}$ IR free potential shift criteria from the natural potential, by using a $1 \mathrm{~mA} / \mathrm{sq} . \mathrm{m}$ current density at the soil resistivity of $3000 \mathrm{ohm}-\mathrm{cm}$ or less, and at higher soil resistivity area, a lower current is expected to be required to provide the required protection [1]. The criterion of $250 \mathrm{mV}$ IR free potential shift criteria was selected based on the above ground surface measurement to ensure that the criteria of $100 \mathrm{mV}$ IR free potential shift could be achieved and guaranteed over circumference of the pipeline. The anode assemblies have been installed in vertically drilled holes on 
both sides of the pipeline at normally 5.5 meters distance from the pipeline's centre line. The anode spacing might vary from groundbed to groundbed, but the average spacing is 13.5 meters for cast zinc (CZ) anode type.

\section{Mathematical model}

The Finite Element Method (FEM) is appropriate for the domains with several media that have finite boundaries. The Boundary Element Method (BEM) is suitable for calculation of the domains which contain only one medium and boundaries that can be finite or infinite. Let's consider the basic principle of the Galerkin's weighted residuals procedure used in the FEM, direct BEM, and iterative sequential Dirichlet-Neumann's hybrid BEM/FEM method. Governing Laplace partial differential equation (PDE) is solved by using the BEM. Integral equation of the steady state current field by using the FEM.

The potentials on the BEM/FEM boundary are calculated by applying the successive underrelaxation iterative method [10].

\subsection{The Finite Element Method (FEM)}

In the FEM, the domain of the considered physical system is divided into finite number of elements of certain geometry called finite elements using so-called procedure discretization of continuum [4-6]. The governing Laplace PDE of the steady state current field is

$$
\frac{\partial}{\partial x}\left(\sigma \frac{\partial \varphi}{\partial x}\right)+\frac{\partial}{\partial y}\left(\sigma \frac{\partial \varphi}{\partial y}\right)+\frac{\partial}{\partial y}\left(\sigma \frac{\partial \varphi}{\partial y}\right)=0
$$

Applying the Galerkin's weighted residuals procedure the solution of electric potentials distribution can be written in the following form:

$$
[\mathrm{H}]^{\mathrm{FEM}} \cdot\{\varphi\}^{\mathrm{FEM}}=\{\mathrm{Q}\}^{\mathrm{FEM}}
$$

where

$[\mathrm{H}]^{\mathrm{FEM}}$ - two-dimensional matrix of coefficients where the common term is given by:

$$
h_{i j}^{F E M}=\sum_{e=1}^{n_{e}}\left[\sigma \int_{V^{e}}\left(\frac{\partial N_{i}^{e}}{\partial x} \frac{\partial N_{j}^{e}}{\partial x}+\frac{\partial N_{i}^{e}}{\partial y} \frac{\partial N_{j}^{e}}{\partial y}+\frac{\partial N_{i}^{e}}{\partial z} \frac{\partial N_{j}^{e}}{\partial z}\right) d V\right]
$$

$\{\Phi\}^{\mathrm{FEM}}$ - column vector matrix of unknown potentials in the nodes of a finite element. Order of this element is $\mathrm{n}_{\mathrm{f}} \mathrm{x} 1$.

$\{\mathrm{Q}\}^{\mathrm{FEM}}$ - column vector matrix of the free terms that contain Neumann boundary conditions. The common term is given by: 


$$
q_{i}^{F E M}=-\sum_{e=1}^{n_{e}}\left[\sum_{j=1}^{n_{f}}\left(\int_{S_{\Delta}^{e}} \sigma N_{i}^{e} \cdot N_{j}^{e} \cdot \frac{\partial \varphi_{j}^{F E M}}{\partial n} d S\right)\right]
$$

$\mathrm{N}_{\mathrm{i}}^{\mathrm{e}}$ - shape functions that are used to approximate unknown potentials function in the following way:

$$
\phi^{F E M}=\sum_{j=1}^{n_{f}} N_{j}^{e} \cdot \phi_{j}^{e}
$$

$\frac{\partial \varphi_{\mathrm{j}}^{\mathrm{FEM}}}{\partial \mathrm{n}}-$ Neumann boundary condition.

\subsection{The direct boundary element method}

The mathematical model of the direct BEM is based on Green's symmetrical identity as well as equations of continuity [3]. These equations serve to apply the boundary conditions on the boundaries between different media. Let's consider two different cases of 3D electrostatic field calculation. The first case is when the observed point $\mathrm{Q}$ falls within the domain $\mathrm{V}$, and the second case is when it falls on the boundary of media.

Generally, formula for potential calculation within, and out of the domain as well as on the boundary is given by:

$$
C(Q) \cdot \varphi(Q)+\int_{S} T(P, Q) \cdot \varphi(P) \cdot d S_{P}=\int_{S} G(P, Q) \cdot \frac{\partial \varphi(P)}{\partial n_{P}} \cdot d S_{P}
$$

where

$G(P, Q)$ - Green function,

$\mathrm{T}(\mathrm{P}, \mathrm{Q})$ - derivative of the Green function in the direction of outward normal vector to the boundary surface,

$\varphi \mathrm{i} \frac{\partial \varphi}{\partial \mathrm{n}}$ - calculated the potential and normal electric field component on the boundary surface

$\mathrm{C}(\mathrm{Q})$ - the constant that can be represented by:

$$
C(Q)= \begin{cases}1 & \text { inside domain } V(\text { Poisson formula }) \\ \frac{1}{2} & \text { on smooth boundary in 3D domain } \\ \frac{\gamma_{3-D}}{4 \pi} & \text { on non - smooth boundary in 3D domain } \\ 0 & \text { outside of domain } V\end{cases}
$$

Applying the collocation point procedure of the weighted residuals method on the equation (6) the solution in the matrix system form can be written: 


$$
[\mathrm{H}]^{\mathrm{BEM}} \cdot\{\varphi\}^{\mathrm{BEM}}=[\mathrm{G}]^{\mathrm{BEM}} \cdot\left\{\frac{\partial \varphi}{\partial \mathrm{n}}\right\}^{\mathrm{BEM}}
$$

where

$[\mathrm{H}]^{\mathrm{BEM}}$ - two-dimensional coefficient matrix with general term defined by:

$$
\begin{array}{r}
\mathrm{h}_{\mathrm{i}, \mathrm{j}}^{\text {BEM }}=\sum_{\mathrm{e}=1}^{\mathrm{n}_{\mathrm{e}}} \int_{\mathrm{S}} \mathrm{N}_{\mathrm{j}}^{\mathrm{e}} \cdot \mathrm{T}_{\mathrm{i}, \mathrm{j}}^{\mathrm{e}} \cdot \mathrm{dS} \mathrm{S}_{\mathrm{p}}+\delta_{\mathrm{i}, \mathrm{j}} \cdot \mathrm{C}_{\mathrm{i}} \\
\left(\mathrm{i}=1,2, . ., \mathrm{n}_{\mathrm{e}} \mathrm{j}=1,2, \ldots, \mathrm{n}_{\mathrm{e}}\right)
\end{array}
$$

$[\mathrm{G}]^{\text {BEM }}$ - two-dimensional coefficient matrix with general term defined by:

$$
\mathrm{g}_{\mathrm{i}, \mathrm{j}}^{\mathrm{BEM}}=\sum_{\mathrm{e}=1}^{\mathrm{n}_{\mathrm{e}}} \int_{\mathrm{S}} \mathrm{N}_{\mathrm{j}}^{\mathrm{e}} \cdot \mathrm{G}_{\mathrm{i}, \mathrm{j}}^{\mathrm{e}} \cdot \mathrm{dS} \quad\left(\mathrm{i}=1,2, . ., \mathrm{n}_{\mathrm{e}} \mathrm{j}=1,2, \ldots, \mathrm{n}_{\mathrm{e}}\right)
$$

$\{\varphi\}^{\mathrm{BEM}} \mathrm{i}\left\{\frac{\partial \varphi}{\partial \mathrm{n}}\right\}^{\mathrm{BEM}}-$ vector column matrix of variables.

The value of variable $\varphi$ or $\partial \varphi / \partial n_{p}$ is known in every single node of the boundary elements for the domain boundary with only one media. So by using the equation (8) the calculation of the variable ( $\varphi$ or $\left.\partial \varphi / \partial n_{p}\right)$ that is not given as a boundary condition ( $\varphi$ or $\partial \varphi / \partial \mathrm{n}_{\mathrm{p}}$ ) is found.

On the boundary between two domains with different media the unknowns are $\varphi$ or $\partial \varphi / \partial \mathrm{n}_{\mathrm{p}}$. In this case separate equation system (6) is formed for each boundary of domain taking into consideration the Dirichlet's and Neumann's boundary conditions. The polarization curve, which describes the relationship between the potential and the current density, indicates the corrosion condition on the pipe surface and is normally used as the boundary condition. It is not an easy task to determine the polarization curve since it strongly depends on a number of phenomena. Furthermore, the polarization curve can also be a function of time and history. One common polarization curve for steel (cathode) can be written in the following form [7]:

$$
i=\frac{A_{\text {pore }}}{A}\left[10^{\frac{V-\phi-E_{F_{e}}}{\beta_{F_{e}}}}-\left(\frac{1}{\left(1-\alpha_{b l k}\right) \cdot i_{\text {lim }, O_{2}}}-10^{\frac{V-\phi-E_{O_{2}}}{\beta_{O_{2}}}}\right)^{-1}-10^{\frac{-\left(V-\phi-E_{H_{2}}\right)}{\beta_{H_{2}}}}\right]
$$

The polarization curve for a galvanic anode would be:

$$
\mathrm{i}=\mathrm{i}_{\mathrm{o}_{2}}\left(10^{\frac{\mathrm{V}-\phi-\mathrm{E}_{\text {corr }}}{\beta_{\text {anode }}}}-1\right)
$$

Where $\mathrm{V}$ is the potential of the steel, obtained from solution of the inner domain, and $\Phi$ is the potential of the soil adjacent to the steel, obtained from solution of the outer domain. The term $\beta_{F e}$ represents the Tafel slope for the corrosion reaction and $E_{F e}$ represents the equilibrium potential for the corrosion reaction [7]. 
On the boundary between two domains with different media additional equations of continuity for $\varphi$ or $\partial \varphi / \partial \mathrm{n}_{\mathrm{p}}$ are written.

\subsection{The hybrid BEM/FEM method}

An example of the cathodic protection system calculation using 3D finite elements discretization of the anodes and cathode is shown on the figure 2 . Discretization of electrolyte using boundary elements as well as metallic connections between anodes and cathode applying 1D finite elements are also shown on the figure 2 .

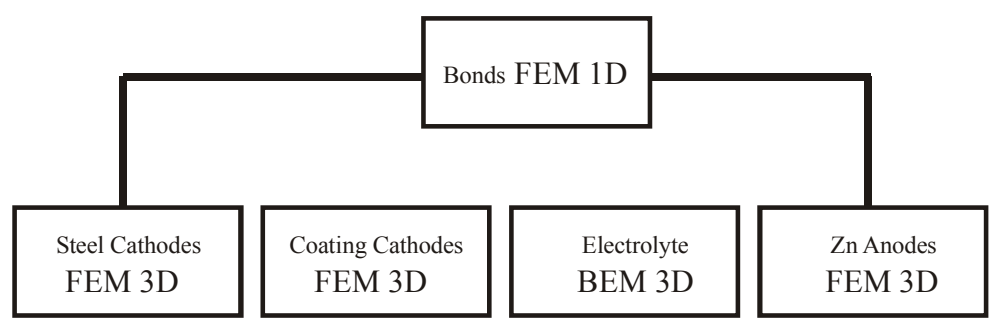

Figure 2: $\quad$ An example of discretization of a cathodic protection system using the coupled BEM/FEM domain.

There are direct and iterative algorithms for linking the finite and boundary element methods. The direct approach requires a system of linear algebraic equations given by

- $\quad$ equation (2) in the FEM domain

- $\quad$ equation (8) in the BEM domain

and the continuity equations for the BEM/FEM boundary.

The significant disadvantage of this approach is the need to solve large system of equations. In order to save the CP memory it is recommended to use some of the following algorithms:

- Robin relaxation coupled algorithm

- Neumann-Neumann coupled algorithm

- Advanced Dirichlet-Neumann algorithm

- Advanced sequential Dirichlet-Neumann algorithm

When using the BEM/FEM iterative method separate calculations for the two separate systems of linear algebraic equations are used, one for the BEM and the other for the FEM domain. The potential distribution or flux, at the BEM/FEM boundary, is then found using the iterative successive underrelaxation method.

\subsection{Advanced sequential Dirichlet-Neumann algorithm}

In this paper advanced sequential Dirichlet-Neumann BEM/FEM algorithm is used. This algorithm consists of the following steps [10]:

- $\quad$ Partition of the whole domain into BEM and FEM domains

- Set initial potential values on the BEM/FEM boundary 
- Start the iterative procedure which lasts until the set convergence condition is fulfilled.

\subsubsection{Calculation of the electric field in BEM domain}

The Dirichlet or Neumann boundary conditions are given on the boundary of the BEM domain, except on the boundary between the BEM and FEM domains. Therefore, for the matrix system (8) in matrices $[\mathrm{H}]^{\mathrm{BEM}}$ and $[\mathrm{G}]^{\mathrm{BEM}}$ the BEMBEM contributions from "pure" BEM boundary and the BEM/FEM contribution from the mutual BEM/FEM boundary could be written in the following form:

$$
[\mathrm{H}]^{\text {BEM }}\left\{\begin{array}{l}
\{\varphi\}_{\mathrm{n}+1}^{\text {BEM-BEM }} \\
\{\varphi\}_{\mathrm{n}+1}^{\text {BEM-FEM }}
\end{array}\right\}=[\mathrm{G}]^{\text {BEM }}\left\{\begin{array}{l}
\left\{\frac{\partial \varphi}{\partial \mathrm{n}}\right\}^{\text {BE+1 }} \\
\left.\left\{\frac{\partial \varphi}{\partial \mathrm{n}}\right\}^{\text {BEM-FEM }}\right\}^{\text {BEM }}
\end{array}\right\}
$$

Also in the system (13) it is necessary to take into account the Dirichlet and Neumann boundary conditions including the potential values at the BEM/FEM boundary from the previous iteration step. Solving the system (13) the normal components of the electric field $\left\{\frac{\partial \varphi}{\partial n}\right\}_{n+1}^{\text {BEM-FEM }}$ at the BEM/FEM boundary will be found.

\subsubsection{Calculation of continuity equation at the BEM/FEM boundary}

$$
\begin{gathered}
\sigma^{\text {FEM }}\left\{\frac{\partial \varphi}{\partial \mathrm{n}}\right\}_{\mathrm{n}+1}^{\text {FEM-BEM }}=-\sigma^{\text {BEM }}\left\{\frac{\partial \varphi}{\partial \mathrm{n}}\right\}_{\mathrm{n}+1}^{\text {BEM-FEM }} \\
\left\{\frac{\partial \varphi}{\partial \mathrm{n}}\right\}_{\mathrm{n}+1}^{\mathrm{FEM}-\mathrm{BEM}}=-\frac{\sigma^{\text {BEM }}}{\sigma^{\text {FEM }}\left\{\frac{\partial \varphi}{\partial \mathrm{n}}\right\}_{\mathrm{n}+1}^{\text {BEM-FEM }}}
\end{gathered}
$$

As a result the Neumann boundary conditions are found that are applicable in the direction of normal vector on the FEM side of the BEM/FEM boundary.

\subsubsection{Calculation of electric field in the FEM domain}

In this step the electric field in the FEM domain is calculated. The Dirichlet or Neumann boundary conditions are specified for the FEM domain boundary except for the FEM-BEM boundary itself. Therefore, for the matrix system (2) in matrices $[\mathrm{H}]^{\mathrm{FEM}}$ the FEM-FEM contributions from "pure" FEM boundary and the FEM/BEM contribution from the mutual FEM/BEM boundary can be written in the following form:

$$
[\mathrm{H}]^{\mathrm{FEM}} \cdot\left\{\begin{array}{l}
\{\varphi\}_{\mathrm{n}+1}^{\mathrm{FEM}-\mathrm{FEM}} \\
\{\varphi\}_{\mathrm{n}+1}^{\mathrm{FEM}-\mathrm{BEM}}
\end{array}\right\}=\left\{\begin{array}{l}
\{\mathrm{Q}\}_{\mathrm{n}+1}^{\mathrm{FEM}-\mathrm{FEM}} \\
\{\mathrm{Q}\}_{\mathrm{n}+1}^{\mathrm{FEM}-\mathrm{BEM}}
\end{array}\right\}
$$

where matrix elements $\{\mathrm{Q}\}_{\mathrm{n}+1}^{\mathrm{FEM}}$ are calculated using expressions (4) and (14). The outcome are potentials $\{\varphi\}_{\mathrm{n}}^{\mathrm{FEM}-\mathrm{BEM}}$ at the FEM/BEM boundary. 


\subsubsection{Correction of calculated potentials at the BEM/FEM boundary}

In this step correction of the potentials calculated in the previous step at the FEM/BEM boundary is done. The correction is made by using the successive underrelaxation method:

$$
\{\varphi\}_{\mathrm{n}+1}^{\text {BEM-FEM }}=(1-\theta) \cdot\{\varphi\}_{\mathrm{n}}^{\text {BEM-FEM }}+\theta \cdot\{\varphi\}_{\mathrm{n}+1}^{\text {FEM-BEM }}
$$

Values of underelaxation $\theta$ are in range $0-1$.

\subsubsection{Check if the convergence criterion is fulfilled}

\section{Calculation results}

The current density distribution over the structure being protected is given in figure 3 . The potential distribution on the ground surface is shown in figure 4 .

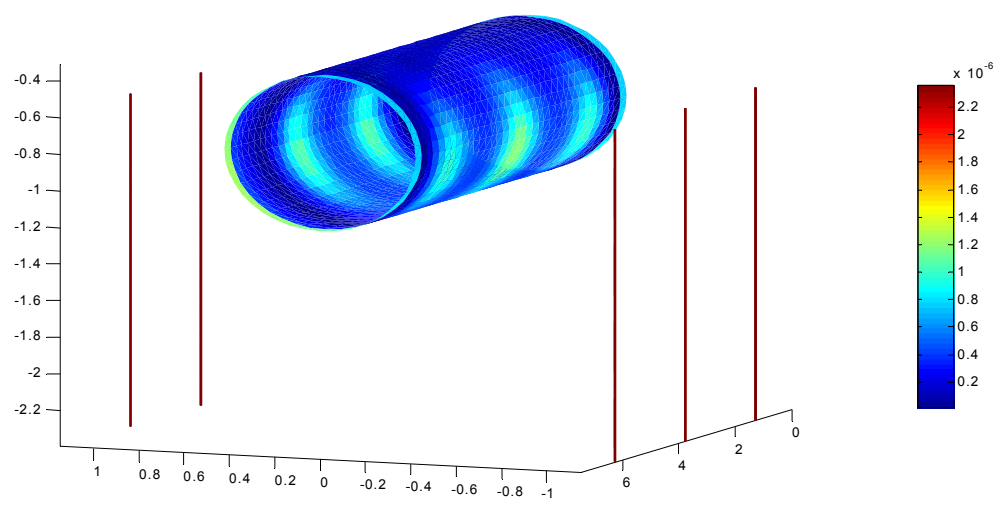

Figure 3: Current density distribution.

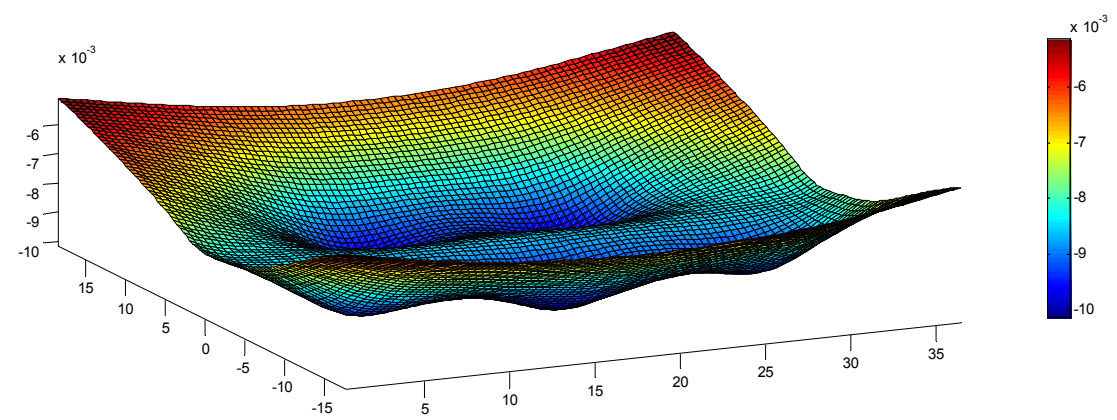

Figure 4: $\quad$ Potential distribution on the ground surface. 


\section{Conclusion}

Application of the coupled BEM/FEM method has proved to be an efficient method for solving the problems in field of cathodic protection. Comparison numerical results have shown good agreement with the readings, protective potentials and current, taken on site.

Also presented algorithm has very stable convergence, and saves on computational time and memory requirements as well. Nonlinear boundary conditions are used in this software. The selection of boundary conditions is very delicate task, and choice of the parameters to be used is subject of serious analysis for specialists in field of electrochemistry.

\section{References}

[1] A. Muharemović, Distribution of the protection current and potential in system of cathodic protection with sacrificial anodes, The $5^{\text {th }}$ Libyancorr. 2005.

[2] A. Muharemović, A contribution to the analysis of the polarization effects in optimization and dimensioning of a cathodic protection system, Sarajevo 1989.

[3] H. Zildžo, The numerical methods in power engineering, ETF Sarajevo 2004.

[4] Haznadar Z., Štih Ž., Electromagnetic fields, waves and numerical methods, Zagreb, 1997.

[5] D. S. Burnett, Finite element analysis from concepts to applications,Addison-Wesley Publishing Company, Massachusetts, 1987.

[6] Zienkiewicz O. C., The Finite Element Method, New York, McGraw-Hill, 1977.

[7] R.A. Adey, (ed). Modelling of Cathodic Protection Systems, Advances in Boundary Elements, Vol. 12, Southampton, Boston, WIT Press 2006.

[8] Kennelley, K.J., Bone, L. \& Orazem, M.E., Current and potential distribution on a coated pipeline with holidays: Part 1. model and experimental verification. Corrosion, 49 (3), pp. 199-210, 1993.

[9] Nisançiöglu, K., Predicting the time dependence of polarization on cathodically protected steel in seawater, Corrosion , 43, pp 100-111, 1987.

[10] Elleithy, W. M., Al-Gahtani, H. J. And El-Gebeily, M., "Iterative Coupling of BE Methods in Elastostatics", Engineering analysis with Boundary Elements, Vol. 25. No. 8, August 2001, pp. 685-695.

[11] H.Zildžo and H. Matoruga, "Calculation of GIS $110 \mathrm{kV}$ Insulating Bushing Apllying Hybrid BEM-FEM Method", XLII. International Scientific Conference on Information, Communication and Energy Systems Technologies - ICES 2007, Ohrid.

[12] H.Zildžo and H. Matoruga, "Calculation of transfered potentials in Substation applied Boundary element methods", 8. Konferenca Slovenskih elektroenergetikov- Sloko CIGRE - Čatež 2007. 